\title{
Habrobracon hebetor and Pteromalus cerealellae as Tools in Post-Harvest Integrated Pest Management
}

\author{
George N. Mbata * and Sanower Warsi \\ Agricultural Research Station, Fort Valley State University, Fort Valley, GA 31030, USA; Sanowerw@yahoo.com \\ * Correspondence: mbatag@fvsu.edu
}

Received: 24 February 2018; Accepted: 23 March 2019; Published: 27 March 2019

\begin{abstract}
Consumers are increasingly demanding pesticide-free grain/legumes and processed foods. Additionally, there are more restrictions, or complete loss, of insecticides labelled for use in managing stored grain insects in post-harvest ecosystems. Suppression of post-harvest pests using parasitic wasps is a more sustainable alternative than chemical pesticides. Habrobracon hebetor (Say) (Hymenoptera: Braconidae) and Pteromalus cerealellae Ashmead (Hymenoptera: Pteromalidae) are two important parasitoids that limit economically important pests of stored products. Host searching ability and reproductive performances of $H$. hebetor and P. cerealellae depend on a wide range of factors, such as host species, commodities, and environmental conditions. Further, use of entomopathogens can complement the ability of parasitoids to regulate pest populations. This review provides information on aspects of $H$. hebetor and P. cerealellae biology and successful regulation of post-harvest pest populations.
\end{abstract}

Keywords: pesticide residue; post-harvest pest; parasitoid; entomopathogens; commodities

\section{Introduction}

Stored product pests cause severe economic losses due to the infestation of commodities in stored grain ecosystems including silos, bakeries, food processing industries, flourmills, and pet food factories. Stored grain managers rely substantially on strategies involving the application of synthetic insecticides to manage stored product pests [1,2]. Although insecticides can be effective, their repeated and indiscriminative use will cause insecticide resistance and detrimental non-target effects, potentially leading to the loss of biodiversity [3]. Insecticide use patterns in post-harvest integrated pest management (IPM) could expose farmers and commodity warehouse workers to acute or chronic exposure to fumigant volatiles [4]. Additionally, consumers could potentially be exposed to pesticide residues in foods [4]. Furthermore, restrictions on insecticide use patterns and increasing consumer demand for pesticide-free foods are catalyzing the search for safe, non-toxic, and sustainable pest management strategies in post-harvest systems [5,6].

Parasitic wasps present an alternative and environmentally compatible approach to overcoming the challenges of synthetic insecticide use in post-harvest systems. Parasitoids do not negatively affect the environment, humans or beneficial organisms. These natural enemies can reproduce continuously for as long as the hosts or alternative hosts are available, thus ensuring sustainability of their populations for long-term regulation of pest populations [7]. Moreover, released parasitoid wasps have the ability to disperse very quickly and locate hosts in hidden corners and crevices in the storage structures $[8,9]$. In addition, potential allergens including insect exuviae and fragments are significantly reduced when parasitoids are used compared with other IPM strategies [10]. The parasitoids Habrobracon hebetor (Say) (Hymenoptera: Braconidae) and Pteromalus cerealellae Ashmead (Hymenoptera: Pteromalidae) are among the important ectoparasitoids of stored product moths and beetles, respectively. 
This paper examines the distribution, life history, host range, behavior, and mode of parasitism of the two parasitoids. The efficacies of the parasitoids in laboratory experiments and field trials were also reviewed. The information provided here will assist in designing IPM programs utilizing biological control.

\section{Distribution}

Habrobracon hebetor and P. cerealellae are cosmopolitan in distribution [11,12]. The taxonomy of $H$. hebetor has been revised several times and this species is also known under the following synonyms: Bracon hebetor Say, Bracon juglandis Ashmead, and Hebrabracon junglandis Ashmead [13]. In the older literature this wasp is commonly called $B$. hebetor. The post-harvest populations of $H$. hebetor fluctuate throughout the year $[14,15]$. While environmental conditions in spring are not particularly conducive to $H$. hebetor, high summer temperatures favor population increases, and wasp populations generally reach maximum numbers in autumn.

Pteromalus cerealellae (Ashmead) has other synonyms including Habroaytus cerealellae (Ashmead 1902) and Pteromalus semota (Walker 1834) [16]. Documented distribution of P. cerealellae extends to Africa, Asia, Caribbean and Europe and North America [16].

\section{Life History and Mating Behavior of Habrobracon hebetor and Pteromalus cerealellae}

Habrobracon hebetor is a small parasitic wasp that is only $2 \mathrm{~mm}$ in length, weighs less than $1 \mathrm{mg}$, is known to have cryptic sibling species that attacks the crop pest Helicoverpa armigera (Hübner), and completes its development in about 9 days when reared on Helicoverpa armigera (Hübner) at $25 \pm 2{ }^{\circ} \mathrm{C}, 65 \pm 5 \% \mathrm{RH}$, and 14:10 (L:D) [17-19]. Female wasps preferably parasitize fourth or fifth instars of moth hosts for egg laying. At $28^{\circ} \mathrm{C}$, female H. hebetor lays 17.46 and 9.64 eggs per day on diapausing and non-diapausing larvae of Plodia interpunctella, respectively [20]. Eggs (0.52 mm length and $0.12 \mathrm{~mm}$ width) of $H$. hebetor are hymenopteriform in shape and are typically attached to paralyzed host larvae. $H$. hebetor has an extremely short duration of development that lasts only 12 days from egg to adult [21,22]. The last instar (2.64 $\mathrm{mm}$ in length, $0.29 \mathrm{~mm}$ head capsule, and $0.95 \mathrm{~mm}$ width) lasts about 4 days before spinning small white cocoons for pupation [21]. The pupal stage lasts about a week [23]. Free-living adult parasitoids begin to search for host larvae to parasitize and lay eggs on immediately after pupation [24]. The mean number of lifetime adult progeny per female is 173.7 in 22 days, with an offspring sex ratio of 1:1 [25]. Male adults have long antennae with 18-20 cylindrical flagellar segments, while females have shorter antennae and are larger in size [26-28].

Habrobracon hebetor displays the arrhenotokous haplo-diploid sex determination mechanism [29,30]. Haploid male offspring develop from unfertilized eggs parthenogenetically, while diploid males or females emerge from fertilized eggs [29]. Females that copulate with diploid males have lower fertility and produce more male-biased broods, compared with those copulated to haploid males [29]. Females avoid mating with males of the same brood; further, newly emerged females have the ability to recognize their brood mates [11,29]. H. hebetor are synovigenic, displaying increased egg production when provisioned diapausing larval P. interpunctella [20]. Female H. hebetor reared on non-diapausing larvae mature more eggs after three days, while those reared on diapausing P. interpunctella larvae mature more eggs from the second day [20]. In addition, oviposition peaks on the third day when $H$. hebetor are reared on diapausing host larvae and on the fourth day if reared on non-diapausing host larvae [20].

Adults of $H$. hebetor are capable of entering photoperiod-induced diapause if exposed to a 10L:14D light regimen at $17.5^{\circ} \mathrm{C}$ or $20^{\circ} \mathrm{C}$ [31]. Adult wasps, particularly those that are diapausing, are less susceptible to low temperatures and can survive at $-5^{\circ} \mathrm{C}$ for up to seven days [31]. Egg and pupal stages of $H$. hebetor had supercooling points below $-25^{\circ} \mathrm{C}$, but no pupae survived up to $12 \mathrm{~d}$ at $-5{ }^{\circ} \mathrm{C}[32]$.

The pteromalid ectoparasitoid P. cerealellae parasitizes a broad host range of pests that develop inside cereal grains and legumes [33]. Adult females oviposit on the late instar of hosts inside the cereal 
kernels or legume seeds $[33,34]$. P. cerealellae eggs are also hymenopteriform in shape with dimensions of $0.54 \times 0.19 \mathrm{~mm}$ [12]. Freshly deposited eggs have a transparent chorion [35] that becomes opaque and white after 24-36 h [35]. Eggs hatch into 13-segmented first instars with a head capsule width that ranges from $0.30 \pm 0.02 \mathrm{~mm}$ to $0.32 \pm 0.01 \mathrm{~mm}$ [12]. P. cerealellae has four larval stages and the larvae have distinct tusk-shaped mandibles, while the pupae and adults have toothed mandibles [35]. Larvae complete development in about 7 days [12]. Adults start to emerge following completion of pupation in 5 days and male adults emerge first [12]. The developmental period from egg to adult at $30 \pm 1{ }^{\circ} \mathrm{C}, 70 \pm 5 \% \mathrm{RH}$, and 12:12 (L:D) h photoperiod lasts about 11 and 12 days for males and females, respectively [35]. Adult male and female $P$. cerealellae can be differentiated based on length of the antennae. The females have longer antennae $(1328 \pm 15.2 \mu \mathrm{m})$ than males $(1308 \pm 21.9 \mu \mathrm{m})$ [12].

\section{Hosts of Habrobracon hebetor and Pteromalus cerealellae}

Habrobracon hebetor mainly attacks larvae of Lepidopteran stored product pests including P. interpunctella, Ephestia kuehniella (Zeller), Ephestia cautella (Walker), Anagasta kuehniella (Zeller), Galleria mellonella (Linnaeus), and Amyelois transitella (Walker) [35]. Some strains of H. hebetor also attack field pests such as H. armigera, Maruca testulalis (Geyer), Spodoptera litura (Fabricius), and Earias vittella (Fabricius) [36].

Pteromalus cerealellae is a parasitoid of internally developing insects including Sitotroga cerealella (Olivier), Callosobruchus maculatus (Fabricius), Lasioderma serricorne (Fabricius), Prostephanus truncatus (Horn), and Sitophilus spp. [34].

\section{Mode of Parasitism of Habrobracon hebetor and Pteromalus cerealellae}

Habrobracon hebetor relies on an insecticidal toxin present in its venom gland for paralyzing the host larvae [17]. The wasp's venom glands are composed of a single layer of eight elongated cells that are connected to the ovipositor [37]. Adult females inject toxin into host larvae by stinging through the cuticle [23]. In H. hebetor, the toxin contains five polypeptides, Brh-I, II, III, IV, and V, with a molecular weight of about $70 \mathrm{kDa}$ [17]. These polypeptides block glutamatergic neuromuscular transmission at the presynaptic nerve terminal, resulting in the death of host larvae within 15 min [38-40]. In addition, the venom of $H$. hebetor also affects other physiological activities of paralyzed host larvae including decline in the production of reactive oxygen species, suppression of phenoloxidase activity in host hemolymph, and reduced encapsulation of hemocytes [41].

Only female parasitoids feed on the hemolymph of paralyzed host larvae during oviposition. Male wasps can feed on artificial diets including diluted honey [37]. Larvae of $H$. hebetor feed and develop on the cuticle of the paralyzed host until pupation [41]. Larvae of $H$. hebetor can switch to another paralyzed host larva for feeding upon the depletion of nutrients in the immediate host larva [37].

While laying eggs, P. cerealellae females inject venom proteins into the host larvae using a stinging apparatus [34]. Injection of venom into the host results in fatal paralysis, which ultimately causes death of the host [34]. Adult and larval stages of P. cerealellae derive nutrients from the host hemolymph that oozes from the punctured integument [34].

\section{Factors Influencing Host Parasitism and Reproductive Efficacies of Habrobracon hebetor and Pteromalus cerealellae}

\subsection{Effect of Host Density on Habrobracon hebetor and Pteromalus cerealellae}

Numerous laboratory studies have reported the effect of host density on the biology of $H$. hebetor [20,42-44]. Generally, parasitism by $H$. hebetor increases with the host densities up to an optimum parasitoid-host ratio [43]. In a previous study by Sanower et al., percentage mortality of non-diapausing host larvae resulting from parasitism by $H$. hebetor decreased beyond an optimum 
parasitoid-host density ratio, but mortality of diapausing larvae was more than $95 \%$ at all host densities investigated [20].

Host density also affects the reproductive efficacy and weight of $F_{1}$ progeny of $H$. hebetor. Egg dispersion by $H$. hebetor on larvae of $P$. interpunctella has been shown to decrease as the host density increased [43]. In a study by Rotary and Gerling, a high host parasitoid ratio increased the percentage egg hatch and adult emergence by H. hebetor when reared on G. mellonella [45]. In a study by Ghimire and Phillips, progeny numbers of $H$. hebetor parasitizing late instars of $P$. interpunctella were higher at high host densities compared to lower densities [44]. The influence of host density on the sex ratio of $H$. hebetor has also been investigated [44-47]. A previous study showed that the offspring sex ratio (male/total) increased with decreasing host/parasitoid ratio [47]. In addition, it has been reported that the egg laying potential of the wasp increased with an increase in host density and this resulted in male biased progeny $[43,48]$.

Host density has been shown to strongly influence the host mortality and progeny of $P$. cerealellae. A previous study showed that the mortality of host larvae, C. maculatus, increased with host density [49]. The attack response of $P$. cerealella to weevil larvae was best described by a type III functional response using an egg limitation model [50]. In this model, the mean attack rate of the parasitoid was estimated to be 1.7 and the upper limit of the response was 24 weevil larvae per individual parasitoid within a 48-h period [50]. The presence of P. cerealella resulted in reduced weight losses of cowpea resulting from weevil infestation [50].

\subsection{Effect of Host Size on Habrobracon hebetor and Pteromalus cerealellae}

Host size is a key factor that influences functional responses of parasitic wasps [51]. H. hebetor has shown a ten-fold higher attraction toward larger mature wandering larvae than small young larvae [16]. In addition, $H$. hebetor prefers larger sized host larvae for egg laying [51]. In a previous study by Mbata et al., daily lifetime fecundity of $H$. hebetor was higher on the larger larvae of $A$. transitella $(55.00 \pm 1.90 \mathrm{mg})$ and G. mellonella $(262.78 \pm 15.17 \mathrm{mg})$ compared to smaller P. interpunctella larvae $(20.15 \pm 0.92 \mathrm{mg})$ [33]. Larger larvae provide better food resources and surface area, which influences oviposition in the wasp $[23,36]$. Furthermore, nutrient-rich diapausing larvae have higher body masses and provide better resources to $H$. hebetor for egg production [20,52].

Host mortality due to parasitism has been shown to vary linearly with increasing host size [53]. It has been reported that the host size influences the reproductive performances of $P$. cerealellae [54]. Larvae of Sitotroga cerealella (Olivier) have been found to be more susceptible to P. cerealellae than those of Sitophilus zeamais and this was ascribed to the larger size of S. cerealella larvae [53]. Weights of male and female offspring of this wasp correlated with the size of host, but the weights of female parasitoid progeny were more influenced by the size of host larvae $[53,54]$.

\subsection{Effect of Host Species, Physiological State of Host, and Maternal Diet of Parasitoid on Progeny of Habrobracon hebetor and Pteromalus cerealellae}

Certain host species enhance reproductive performance of wasps better than others. For example, $H$. hebetor has been shown to perform better on stored product moths, such as E. kuehniella and P. interpunctella, than on crop pests including H. armigera and Malacosoma disstria Hübner (Lepidoptera: Lasiocampidae) [36,55].

Smith et al. showed that rearing P. cerealella on S. cerealellae resulted in higher progeny numbers compared with rearing the parasitoid on S. zeamais [53]. S. cerealellae was found to be highly susceptible to $P$. cerealellae when parent parasitoids were also reared on $S$. cerealellae, but when parent parasitoids were reared on S. zeamais, parasitism of S. cerealellae decreased [53].

The physiological state of the host has been shown to affect the fecundity and weight of parasitoid offspring [20]. H. hebetor reared on diapausing P. interpunctella larvae produced more offspring, which were heavier than offspring from parent parasitoids that were fed on diapausing host larvae. 
Progeny production, development, and foraging behavior of $H$. hebetor are strongly affected by the diet of the host [56]. A study on the fecundity, developmental period, longevity of adults, and offspring sex ratio of $H$. hebetor on the larvae of E. kuehniella raised on four different diets (rice flour, corn flour, wheat flour, and barley flour) demonstrated that the highest number of $F_{1}$ females was recorded when the host diet was rice flour [56]. However, the total number of adult progeny was significantly higher when $H$. hebetor was reared on the larvae of E. kuehniella fed on wheat flour combined with $20 \%$ glycerol diet [57]. Conversely, a lower number of progeny was obtained when the host diet was whole-wheat flour only [57].

Supplementing parasitoid hosts with sugar enhanced the survival of $P$. cerealellae and resulted in a female-biased sex ratio of offspring [58]. However, the longevity of female wasps was higher than those of the males, irrespective of whether parent parasitoids were provisioned with sugar supplement or not. Lifetime fecundity of $P$. cerealellae was not affected by diet [58].

\subsection{Effect of Available Space on Efficiency of Habrobracon hebetor and Pteromalus cerealellae}

The results of container size on the efficacy of parasitoids with respect to the mortality of hosts have not been consistent. In a laboratory experiment, the proportion of paralyzed larvae did not vary remarkably in response to container size $\left(0.0001672\right.$ to $\left.0.00038 \mathrm{~m}^{3}\right)$, and more than $90 \%$ of host larvae (diapausing and non-diapausing larvae of P. interpunctella) were parasitized by H. hebetor [59]. However, parasitization of E. kuehniella decreased as the volume of storage structures scaled up from 0.006415 to $8 \mathrm{~m}^{3}$ [60]. In another study, the mortality of P. interpunctella larvae exposed to H. hebetor in a cage $\left(0.0029 \mathrm{~m}^{3}\right)$ and a storage house $\left(25-32 \mathrm{~m}^{3}\right)$ also demonstrated that the mortality of hosts decreased as available storage space increased [61].

In a previous study by Mbata et al., mortality of $C$. maculatus larvae due to parasitism by $P$. cerealellae was significantly higher in small containers of about $5 \mathrm{~cm}^{3}$ compared with larger containers [49]. However, mortality was not significantly different in containers that ranged in size from 57.4 to $202.4 \mathrm{~cm}^{3}$ [49]. The spacing between seeds likely played a role in the differences in mortality [49]. It is probable that different parasitoids will have different search distances, and this is likely to influence parasitoid efficacy in large storage structures. The numbers of parasitoid progeny were not affected by size of storage structures. In previous studies, the numbers of adult progeny of $H$. hebetor were not different when the sizes of rearing containers were varied $[20,44]$ and the results were consistent when P. interpunctella, E. kuehniella, or the combination of both species were offered to H. hebetor [51].

\subsection{Effect of Semiochemicals on the Recruitment of Habrobracon hebetor and Pteromalus cerealellae to} Storage Containers

The potential role of semiochemicals in the location of hosts or host habitats by parasitoids of the families Braconidae and Pteromalidae have been studied [33,62]. Volatile compounds emitted by the host, or host-related odor sources, such as feces, frass, debris, host habitat, or food, were identified as the stimuli eliciting the strongest attractions to female parasitoids of $H$. hebetor and P. cerealellae [33,62-64]. Stimuli have been identified to elicit short- and long-range responses by the parasitoids [62]. The stimuli that elicit long-range responses recruit parasitoids to the habitat of the hosts, while short-range responses lead the parasitoids to the host. Some authors hypothesize that integrating these semiochemicals with parasitoid mass release programs could enhance parasitoid efficiency [62].

\subsection{Effect of Age on the Fecundity of Habrobracon hebetor and Pteromalus cerealellae}

Parasitoid age is a critical factor influencing the reproductive performances of $H$. hebetor [65]. Older females of $H$. hebetor (10 days old) have been shown to produce fewer progeny when reared on E. kuehniella and G. mellonella; progeny reduction when reared on E. kuehniella or G. mellonella was 34\% 
and 26.7\%, respectively [65]. Male-biases of parasitoid offspring became more pronounced with older parasitoids, irrespective of whether they were reared on E. kuehniella or G. mellonella [65].

With respect to $P$. cerealellae, progeny production has been shown to be significantly affected by the parasitoid age [55]. In a study by Onagbola et al., provisioning with supplemental food, such as glucose for $P$. cerealellae females, averted the effect of aging on oviposition, as 16-20-day-old female parasitoids that were fed on sugar solution produced significantly more progeny than starved females of the same age group [55].

\subsection{Influence of Temperature on Habrobracon hebetor and Pteromalus cerealellae}

Host parasitism and life history traits of $H$. hebetor are affected by abiotic stresses including temperature, moisture, and light [66,67]. Foraging behavior and reproductive performance of $H$. hebetor are affected by temperature inside storage facilities [68-70]. Egg production by the Braconid ectoparasitoid has been shown to increase with temperature and maximum fecundity occurred at $25{ }^{\circ} \mathrm{C}$ [68], while egg laying capacity was observed to decline at cold temperatures of 3 and $5{ }^{\circ} \mathrm{C}$ [71-73]. In addition, cooler storage temperatures have the potential to decrease parasitism, resulting in a decrease in host mortality [72]. Though the developmental time of immature stages of $H$. hebetor have been shown to decrease with increases in temperature [72], the lower threshold observed for development was between $11-12{ }^{\circ} \mathrm{C}$ [74]. The larval stages of $H$. hebetor are very sensitive to temperature. $H$. hebetor can survive and complete its life cycle within a temperature range of $15-40{ }^{\circ} \mathrm{C}$ [75]. The sex ratio of $H$. hebetor is affected by temperature and exposure time [69]. For instance, the sex ratio of $H$. hebetor offspring skewed towards males when parents were kept at 3 or $5^{\circ} \mathrm{C}$ for 4 or 3 weeks, respectively [72].

\section{Combined Application of Parasitoids and Other Biocontrol Agents}

\subsection{Combination of Parasitoid Species}

In a study by Castañé et al., Habrobracon hebetor was able to affect 30\% and $40 \%$ mortality of E. kuehniella and P. interpunctella in small storage experiments; interestingly, combining H. hebetor and Venturia canescens (Gravenhorst) (Hymenoptera: Ichneumonidae) against the same hosts in the same system did not generate host mortalities that were significantly higher than the application of $H$. hebetor alone [61].

\subsection{Combination of Habrobracon hebetor and Bacillus thuringiensis}

A study showed that combined application of H. hebetor and Bacillus thuringiensis (Bt) caused $86 \%$ mortality of the exposed P. interpunctella larvae, while deploying $H$. hebetor and Bt separately resulted in about $35 \%$ and $42 \%$ mortality, respectively [76]. It has been reported that the efficiency of $H$. hebetor as a parasitoid increased when the wasp was cultured with Bt [77]. For instance, $64 \%, 66 \%$, and $73 \%$ mortality rates of Corcyra cephalonica (Lepidoptera: Gelleridae) (Stainton) larvae were obtained when $H$. hebetor was reared on a diet fortified with $\mathrm{LC}_{25}, \mathrm{LC}_{10}$, and $\mathrm{LC}_{50}$ of $\mathrm{Bt}$, respectively. Conversely, wasps reared without Bt resulted in 37\% mortality of host larvae [77]. In addition, synergy has been demonstrated between $\mathrm{Bt}$ and $\mathrm{H}$. hebetor in the management of the field pest Sodoptera littoralis Boisd (Lepidoptera: Noctuidae) [78]. However, interaction between the wasp and Bt reduced the oviposition and progeny size of the parasitoid [73].

\subsection{Habrobracon hebetor and Entomopathogenic Nematodes (EPNs)}

Entomopathogenic nematodes of the families of Steinernematidae and Heterorhabditidae have been studied extensively for the management of a wide range of stored product pests [79]. Entomopathogens have the potential to be incorporated into biological control strategies of post-harvest IPM programs. Nematodes parasitize host larvae through their mutualistic relationship with bacteria (Xenorhabdus for Steinernematidae and Photorhabdus for Heterorhabditidae) that inhabit the intestine 
of the nematodes [80]. The infective juvenile (IJ) nematodes enter the hemocoel of the hosts through natural apertures including the mouth, anus, or spiracles and release pathogenic bacteria that multiply and kill the hosts within $48 \mathrm{~h}$ [80].

Larvae of $P$. interpunctella are highly susceptible to the combined application of Heterorhabditis indica IJs and the parasitoid H. hebetor. The interaction between the two has been reported to result in either additive or synergistic increases in the mortality of P. interpunctella [80]. Nematode-parasitoid interaction had no significant effect on the survival of adult and pupal parasitoids but affected the survival of the parasitoid larvae, suggesting that proper timing of the application of IJs of EPN and parasitoid release could circumvent the impact the nematodes could have on the larvae of parasitoids [80]. Moreover, it has also been noted that other parasitoids have been integrated with entomopathogenic nematodes for successful management of post-harvest pests [81].

\subsection{Integration of Habrobracon hebetor with Entomopathogenic Fungi}

Entomopathogenic fungi have the potential to contribute significantly to the suppression of stored insect pests. These fungi have also been investigated for integration with parasitoids [82-84]. Strains of Beauveria bassiana and Metarhizium anisopliae have been found to be pathogenic to larvae of $H$. hebetor, but not the pupae [84]. However, some isolates of B. bassiana (EUT105) and M. anisopliae (M-396) did not show adverse effects on the larval and pupal stages of H. hebetor [84]. Stinging by H. hebetor has been shown to increase the susceptibility of $G$. mellonella to B. bassiana, because the H. hebetor attack increased the conidial germination of $B$. bassiana on host larvae resulting in reduced phenoloxidase (PO) activity and hemocyte encapsulation rate in G. mellonella larvae $[85,86]$. These entomopathogenic fungi strains could therefore be integrated with parasitic wasps in the management of stored product pests.

\section{Commercial Applications of Parasitoids and Integration with Other Natural Enemies}

A few studies have demonstrated the potential of parasitoid wasps in the management of post-harvest pests in commercial warehouses. For example, H. hebetor occurs naturally and was found with other hymenopteran wasps on E. kuehniella-infested wheat in Italy and was also reported in fig storage warehouses in California and Greece [87-89]. The use of H. hebetor with Trichogramma evanescens Westwood (Hymenoptera: Trichogrammatidae) against E. kuehniella and P. interpunctella in organic bakeries and mills in Germany and Austria has been demonstrated to be effective [90]. The combined use of $H$. hebetor and mating disruption (MD) to manage P. interpunctella populations in a chocolate factory (about $2000 \mathrm{~m}^{2}$ area and $5 \mathrm{~m}$ height) decreased moths captured on traps [91]. In a peanut stock warehouse, the release of $H$. hebetor alone resulted in the $66.1 \%$ and $97.3 \%$ reduction of P. interpunctella and C. cautella, respectively [92]. Combining H. hebetor with Trichogramma pretiosum increased mortality of $P$. interpunctella to $84.0 \%$ and that of C. cautella to $98.0 \%$ [92].

\section{Conclusions}

Extensive information is currently available regarding foraging behavior and functional responses of parasitoids to facilitate implementation of bio-rational integrated pest management strategies in post-harvest systems. These parasitoids should be deployed in the management of post-harvest pests in the organic food industries, especially when a few insect fragments could be tolerated. The integration of parasitoids and complimentary IPM tools can be deployed in the management of residual populations of stored product pests in warehouses and other grain or processed food storage structures. H. hebetor and P. cerealellae are cosmopolitan in distribution and well suited to a range of climatic and environmental conditions. Their integration into pest management can and should be more broadly implemented.

Acknowledgments: The authors are thankful to Michael Toews, Department of Entomology, University of Georgia, Tifton, GA, USA, for painstakingly editing this manuscript.

Conflicts of Interest: The authors declare no conflict of interest. 


\section{References}

1. El-Aziz, S.E. Control strategies of stored product pests. J. Entomol. 2011, 8, 101-122.

2. Dubey, N.K.; Srivastava, B.; Kumar, A. Current status of plant products as botanical pesticides in storage pest management. J. Biopestic. 2008, 1, 182-186.

3. Champ, B.R.; Dyte, C.E. FAO global survey of pesticide susceptibility of stored grain pests. FAO Plant Prot. Bull. 1977, 25, 49-67.

4. Damalas, C.A.; Eleftherohorinos, I.G. Pesticide exposure, safety issues, and risk assessment indicators. Int. J. Environ. Res. Public Health 2011, 8, 1402-1419. [CrossRef]

5. Wesseling, C.; Corriols, M.; Bravo, V. Acute pesticide poisoning and pesticide registration in Central America. Toxicol. Appl. Pharmacol. 2005, 207, 697-705. [CrossRef]

6. Spadaro, D.; Gullino, M.L. State of the art and future prospects of the biological control of post-harvest fruit diseases. Int. J. Food Microbial. 2004, 91, 185-194. [CrossRef]

7. Ueno, T.; Ueno, K. The effects of host-feeding on synovigenic egg development in an endoparasitic wasp, Itoplectis naranyae. J. Insect Sci. 2007, 7, 46. [CrossRef] [PubMed]

8. Quicke, D.L.; Fitton, M.G. Ovipositor steering mechanisms in parasitic wasps of the families Gasteruptiidae and Aulacidae (Hymenoptera). Proc. R. Soc. Lond. B 1995, 261, 99-103.

9. Wang, X.; Yang, Z. Behavioral mechanisms of parasitic wasps for searching concealed insect hosts. Acta Ecol. Sin. 2008, 28, 1257-1269.

10. Flinn, P.W.; Hagstrum, D.W. Augmentative releases of parasitoid wasps in stored wheat reduces insect fragments in flour. J. Stored Prod. Res. 2001, 37, 179-186. [CrossRef]

11. Ode, P.J.; Antolin, M.F.; Strand, M.R. Brood-mate avoidance in the parasitic wasp Bracon hebetor Say. Anim. Behav. 1995, 49, 1239-1248. [CrossRef]

12. Onagbola, E. Studies on the Biology and host Location Behavior of Pteromalus cerealellae (Ashmead) (Hymenoptera: Pteromalidae), a Parasitoid of Callosobruchus maculatus (F.) (Coleoptera: Chrysomelidae). Ph.D. Thesis, Auburn University, Auburn, AL, USA, 2008.

13. Krombrien, P.D.; Hurd, P.D.; Smith, D.R.; Burks, B.D. Catalog of Hymenoptera in America North of Mexico Vol. I; Smithsonian Institution Press: Washington, DC, USA, 1979.

14. Antolin, M.F.; Strand, M.R. Mating system of Bracon hebetor (Hymenoptera: Braconidae). Ecol. Entomol. 1992, 17, 1-7. [CrossRef]

15. Ode, P.J.; Antolin, M.F.; Strand, M.R. Constrained oviposition and female-biased sex allocation in a parasitic wasp. Oecologia 1997, 109, 547-555. [CrossRef] [PubMed]

16. Hagstrum, D.W.; Subramanyam, B. Stored-Product Insect Resource; Woodhead Publishing and AACC International Press: St. Paul, MN, USA, 2016.

17. Quistad, G.B.; Leisy, D.J. Insecticidal Toxins from the parasitic wasp, Bracon hebetor. U.S. Patent US5554592A, 10 September 1996.

18. Saxena, H.; Ponnusamy, D.; Asif Iquebal, M. Seasonal parasitism and biological characteristics of Habrobracon hebetor (Hymenoptera: Braconidae) - A potential larval ectoparasitoid of Helicoverpa armigera (Lepidoptera: Noctuidae) in a chickpea ecosystem. Biocontrol. Sci. Technol. 2012, 22, 305-318. [CrossRef]

19. Heimpel, G.E.; Antolin, M.F.; Franqui, R.A.; Strand, M.R. Reproductive isolation and genetic variation between two strains of Bracon hebetor (Hymenoptera: Braconidae). Biol. Control 1997, 9, 149-156. [CrossRef]

20. Sanower, W.; Mbata, G.N.; Payton, M.E. Improvement of reproductive performance of Habrobracon hebetor: Consideration of diapausing and non-diapausing larvae of Plodia interpunctella. Biol. Control 2017, 118, 32-36. [CrossRef]

21. Pezzini, C.; Jahnke, S.M.; Köhler, A. Morphological characterization of immature stages of Habrobracon hebetor (Hymenoptera, Braconidae) ectoparasitoid of Ephestia kuehniella (Lepidoptera, Pyralidae). J. Hymenopt. Res. 2017, 60, 157-172. [CrossRef]

22. Saadat, D.; Bandani, A.R.; Dastranj, M. Comparison of the developmental time of Bracon hebetor (Hymenoptera: Braconidae) reared on five different lepidopteran host species and its relationship with digestive enzymes. Eur. J. Entomol. 2014, 111, 495-500. [CrossRef]

23. Ghimire, M.N. Reproductive Performance of the Parasitoid Bracon hebetor Say (Hymenoptera: Braconidae) on Various Host Species of Lepidoptera. Ph.D. Thesis, Oklahoma State University, Stillwater, OK, USA, 2008. 
24. Desouhant, E.; Driessen, G.; Amat, I.; Bernstein, C. Host and food searching in a parasitic wasp Venturia canescens: A trade-off between current and future reproduction? Anim. Behav. 2005, 70, 145-152. [CrossRef]

25. Youm, O.; Gilstrap, F.E. Life-fertility tables of Bracon hebetor Say (Hymenoptera: Braconidae) reared on Heliocheilus albipunctella de Joannis (Lepidoptera: Noctuidae). Int. J. Trop. Insect Sci. 1993, 14, 455-459. [CrossRef]

26. Abbott, B.D.; Grosch, D.S. Antennal bud development in Bracon hebetor (Hymenoptera: Braconidae) examined by light and electron microscopy during the third and fourth instars. Ann. Entomol. Soc. Am. 1987, 80, 353-360. [CrossRef]

27. Dweck, H.K.; Gadallah, N.S. Description of the antennal sensilla of Habrobracon hebetor. BioControl 2008, 53, 841-856. [CrossRef]

28. Harvey, J.A.; Strand, M.R. Sexual size and development time dimorphism in a parasitoid wasp: An exception to the rule? Eur. J. Entomol. 2003, 100, 485-492. [CrossRef]

29. Antolin, M.F.; Ode, P.J.; Heimpel, G.E.; O'Hara, R.B.; Strand, M.R. Population structure, mating system, and sex-determining allele diversity of the parasitoid wasp Habrobracon hebetor. Heredity 2003, 91, 373-381. [CrossRef]

30. Altuntaş, H.; Kilic, A.Y.; Sivas, H. The effects of parasitism by the ectoparasitoid Bracon hebetor Say (Hymenoptera: Braconidae) on host hemolymph proteins in the Mediterranean flour moth Ephestia kuehniella Zeller (Lepidoptera: Pyralidae). Turk. J. Zool. 2010, 34, 409-416.

31. Chen, H.; Zhang, H.; Zhu, K.Y.; Throne, J.E. Induction of reproductive diapause in Habrobracon hebetor (Hymenoptera: Braconidae) when reared at different photoperiods at low temperatures. Environ. Entomol. 2012, 41, 697-705. [CrossRef]

32. Carrillo, M.A.; Heimpel, G.E.; Moon, R.D.; Cannon, C.A.; Hutchison, W.D. Cold hardiness of Habrobracon hebetor (Say) (Hymenoptera: Braconidae), a parasitoid of pyralid moths. J. Insect Physiol. 2005, 51, 759-768. [CrossRef]

33. Mbata, G.N.; Shu, S.; Phillips, T.W.; Ramaswamy, S.B. Semiochemical cues used by Pteromalus cerealellae (Hymenoptera: Pteromalidae) to locate its host, Callosobruchus maculatus (Coleoptera: Bruchidae). Ann. Entomol. Soc. Am. 2004, 97, 353-360. [CrossRef]

34. Onagbola, E.O.; Fadamiro, H.Y. Assessment of frozen larvae of Callosobruchus maculatus as hosts for rearing Pteromalus cerealellae (Ashmead) (Hymenoptera: Pteromalidae). Biol. Control 2009, 48, 36-41. [CrossRef]

35. Ghimire, M.N.; Phillips, T.W. Oviposition and reproductive performance of Habrobracon hebetor (Hymenoptera: Braconidae) on six different pyralid host species. Ann. Entomol. Soc. Am. 2014, 107, 809-817. [CrossRef]

36. Dabhi, M.R.; Korat, D.M.; Vaishnav, P.R. Comparative biology of Bracon hebetor Say on seven lepidopteran hosts. Karnataka J. Agric. Sci. 2012, 24, 549-550.

37. Beard, R.L. Toxicology of Habrobracon venom: A study of natural insecticides. Conn. Agric. Exp. Stn. New Haven Bull. 1952, 562, 1-27.

38. Hagstrum, D.W.; Smittle, B.J. Host utilization by Bracon hebetor. Environ. Entomol. 1978, 7, 596-600. [CrossRef]

39. Usherwood, P.T.; Machili, P. Chemical transmission at the insect excitatory neuromuscular synapse. Nature 1966, 210, 634-636. [CrossRef] [PubMed]

40. Walther, C.; Rathmayer, W. The effect of Habrobracon venom on excitatory neuromuscular transmission in insects. J. Comp. Physiol. A Neuroethol. Sens. Neural Behav. Physiol. 1974, 89, 23-38.

41. Kryukova, N.A.; Dubovskiy, I.M.; Chertkova, E.A.; Vorontsova, Y.L.; Slepneva, I.A.; Glupov, V.V. The effect of Habrobracon hebetor venom on the activity of the prophenoloxidase system, the generation of reactive oxygen species and encapsulation in the haemolymph of Galleria mellonella larvae. J. Insect Physiol. 2011, 57, 796-800. [CrossRef] [PubMed]

42. Eliopoulos, P.A.; Stathas, G.J. Life tables of Habrobracon hebetor (Hymenoptera: Braconidae) parasitizing Anagasta kuehniella and Plodia interpunctella (Lepidoptera: Pyralidae): Effect of host density. J. Econ. Entomol. 2008, 101, 982-988. [CrossRef] [PubMed]

43. Yu, S.H.; Ryoo, M.I.; Na, J.H.; Choi, W.I. Effect of host density on egg dispersion and the sex ratio of progeny of Bracon hebetor (Hymenoptera: Braconidae). J. Stored Prod. Res. 2003, 39, 385-393. [CrossRef]

44. Ghimire, M.N.; Phillips, T.W. Mass rearing of Habrobracon hebetor Say (Hymenoptera: Braconidae) on larvae of the Indian meal moth, Plodia interpunctella (Lepidoptera: Pyralidae): Effects of host density, parasitoid density, and rearing containers. J. Stored Prod. Res. 2010, 46, 214-220. [CrossRef] 
45. Alam, M.; Alam, S.; Miah, M.; Mian, M.; Hossain, M. Mass rearing of Bracon hebetor (Hym.: Braconidae) on wax moth, Galleria mellonella (Lep.: Pyralidae) with varying density of parasitoid and the host. J. Crop Prot. 2015, 5, 39-48. [CrossRef]

46. Rotary, N.; Gerling, D. The influence of some external factors upon the sex ratio of Bracon hebetor Say (Hymenoptera: Braconidae). Environ. Entomol. 1973, 2, 134-138. [CrossRef]

47. Benson, J.F. Intraspecific competition in the population dynamics of Bracon hebetor Say (Hymenoptera: Braconidae). J. Anim. Ecol. 1973, 42, 105-124. [CrossRef]

48. Jamil, A.; Ul Abdin, Z.; Arshad, M.; Falabella, P.; Abbas, S.K.; Tahir, M.; Jamil, A.; Manzoor, A.; Shaina, H. Multiple effects of host density on egg density and the sex ratio of progeny of Bracon hebetor (Say.) (Hymenoptera: Braconidae). Pak. J. Zool. 2015, 47, 455-460.

49. Mbata, G.N.; Thomas, A.; Fadamiro, H.F. Parasitism by Pteromalus cerealellae (Hymenoptera: Pteromalidae) on the Cowpea weevil, Callosbruchus maculatus (Coleoptera: Bruchidae): Host density, temperature effects, and host finding ability. Biol. Control 2005, 33, 286-292. [CrossRef]

50. Mbata, G.N.; Brewster, C.C. Functional response of Pteromalus cerealellae (Hymenoptera: Pteromalidae) on the cowpea weevil, Callosbruchus maculatus (Coleoptera: Bruchidae), and interaction between parasitism and cowpea varietal susceptibility. Biocontrol 2009, 54, 751-761. [CrossRef]

51. Akinkurolere, R.O.; Boyer, S.; Chen, H.; Zhang, H. Parasitism and host-location preference in Habrobracon hebetor (Hymenoptera: Braconidae): Role of refuge, choice, and host instar. J. Econ. Entomol. 2009, 102, 610-615. [CrossRef] [PubMed]

52. Mbata, G.N.; Pascual-Villalobos, M.J.; Payton, M.E. Comparative mortality of diapausing and nondiapausing larvae of Plodia interpunctella (Lepidoptera: Pyralidae) exposed to monoterpenoids and low pressure. J. Econ. Entomol. 2012, 105, 679-685. [CrossRef] [PubMed]

53. Smith, L.; Weaver, D.K.; Arbogast, R.T. Suitability of the maize weevil and angoumois grain moth as hosts for the parasitoids Anisopteromalus calandrae and Pteromalus cerealellae. Entomol. Exp. Appl. 1995, 76, 171-177. [CrossRef]

54. Wen, B.; Weaver, D.K.; Brower, J.H. Size preference and sex ratio for Pteromalus cerealellae (Hymenoptera: Pteromalidae) parasitizing Sitotroga cerealella (Lepidoptera: Gelechiidae) in stored corn. Environ. Entomol. 1995, 24, 1160-1166. [CrossRef]

55. Rafiee, D.H.; Hejazi, M.J.; Nouri, G.G.; Saber, M. Toxicity of some biorational and conventional insecticides to cotton bollworm, Helicoverpa armigera (Le-pidoptera: Noctuidae) and its ectoparasitoid, Habrobracon hebetor (Hymenoptera: Braconidae). J. Entomol. Soc. Iran 2008, 28, 27-73.

56. Faal, M.A.H.; Shishehbor, P. Biological parameters of Bracon hebetor (Hymenoptera: Braconidae) parasitizing Ephestia kuehniella (Lepidoptera: Pyralidae): Effect of host diet. J. Crop Prot. 2013, 2, 411-419.

57. Eslampour, L.; Aramideh, S. Adult longevity, fertility and sex ratio of Habrobracon hebetor (Say) (Hymenoptera: Braconidae) parasitizing Ephestia kuehniella (Zeller) (Lepidoptera: Pyralidae): Effect of host artificial diets. J. Entomol. Zool. Stud. 2016, 4, 189-192.

58. Onagbola, E.O.; Fadamiro, H.Y.; Mbata, G.N. Longevity, fecundity, and progeny sex ratio of Pteromalus cerealellae in relation to diet, host provision, and mating. Biol. Control 2007, 40, 222-229. [CrossRef]

59. Warsi, S.; Mbata, G.N. Impact of peanut depth and container size on the parasitism of diapausing and nondiapausing larvae of Indian Meal Moth (Lepidoptera: Pyralidae) by Habrobracon hebetor (Hymenoptera: Braconidae). Environ. Entomol. 2018, 47, 1226-1232. [CrossRef] [PubMed]

60. Paust, A.; Reichmuth, C.; Büttner, C.; Prozell, S.; Adler, C.; Schöller, M. Spatial effects on competition between the larval parasitoids Habrobracon hebetor (Say) (Hymenoptera: Braconidae) and Venturia canescens (Gravenhorst) (Hymenoptera: Ichneumonidae) parasitising the Mediterranean flour moth, Ephestia kuehniella Zeller (Lepidoptera: Pyralidae). In Proceedings of the 9th International Working Conference on Stored Product Protection, Campinas, São Paulo, Brazil, 15-18 October 2006; pp. 797-803.

61. Castañé, C.; Riudavets, J.; Lucas, E. Parasitism of single or combined pyralid populations by Venturia canescens and Habrobracon hebetor in laboratory and storeroom conditions. J. Pest Sci. 2018, 91, 1421-1428. [CrossRef]

62. Mbata, G.N.; Eason, J.; Payton, M.E.; Davis, M.F. Putative host volatiles used by Habrobracon hebetor (Hymenoptera: Braconidae) to locate larvae of Plodia interpunctella (Lepidoptera: Pyralidae). J. Insect Behav. 2017, 30, 287-299. [CrossRef] 
63. Vet, L.E.; Groenewold, A.W. Semiochemicals and learning in parasitoids. J. Chem. Ecol. 1990, 16, 3119-3135. [CrossRef]

64. Blassioli-Moraes, M.C.; Borges, M.; Michereff, M.F.F.; Magalhães, D.M.; Laumann, R.A. Semiochemicals from plants and insects on the foraging behavior of Platygastridae egg parasitoids. Pesquisa Agropecuária Bras. 2016, 51, 454-464. [CrossRef]

65. Gündüz, E.A.; Gülel, A. Investigation of fecundity and sex ratio in the parasitoid Bracon hebetor Say (Hymenoptera: Braconidae) in relation to parasitoid age. Turk. J. Zool. 2005, 29, 291-294.

66. Abram, P.K.; Cusumano, A.; Peri, E.; Brodeur, J.; Boivin, G.; Colazza, S. Thermal stress affects patch time allocation by preventing forgetting in a parasitoid wasp. Behav. Ecol. 2015, 26, 1326-1334. [CrossRef]

67. Huey, R.B.; Kingsolver, J.G. Evolution of thermal sensitivity of ectotherm performance. Trends Ecol. Evol. 1989, 4, 131-135. [CrossRef]

68. Asl, M.H.A.; Talebi, A.A.; Kamali, H.; Kazemi, S. Stored product pests and their parasitoid wasps in Mashhad, Iran. Adv. Environ. Biol. 2009, 3, 239-243.

69. Arthur, F.H.; Hagstrum, D.W.; Flinn, P.W.; Reed, C.R.; Phillips, T.W. Insect populations in grain residues associated with commercial Kansas grain elevators. J. Stored Prod. Res. 2006, 42, 226-239. [CrossRef]

70. Puttarudriah, M.; Basavanna, G.C. A study on the identity of Bracon hebetor Say and Bracon brevicornis Wesmael (Hymenoptera: Braconidae). Bull. Entomol. Res. 1956, 47, 183-191. [CrossRef]

71. Ahmed, M.S.H.; Al-Maliky, S.K.; Al-Taweel, A.A.; Jabo, N.F.; Al-Hakkak, Z.S. Effects of three temperature regimes on rearing and biological activities of Bracon hebetor (Say) (Hymenoptera: Braconidae). J. Stored Prod. Res. 1985, 21, 65-68. [CrossRef]

72. Askari Seyahooei, M.; Mohammadi-Rad, A.; Hesami, S.; Bagheri, A. Temperature and exposure time in cold storage reshape parasitic performance of Habrobracon hebetor (Hymenoptera: Braconidae). J. Econ. Entomol. 2018, 111, 564-569. [CrossRef] [PubMed]

73. Forouzan, M.; Amir, M.M.; Sahragard, A. Temperature-dependent development of Habrobracon hebetor (Hym.: Braconidae) reared on larvae of Galleria mellonella (Lep.: Pyralidae). J. Entomol. Soc. Iran. 2008, 28, 67-78.

74. Golizadeh, A.; Alikhani, M.; Hassanpour, M.; Enkegaard, A.; Rafiee-Dastjerdi, H.; Razmjou, J. Comparative biology and life table of Habrobracon hebetor (Hymenoptera: Braconidae) on Anagasta kuehniella (Lepidoptera: Pyralidae) at five constant temperatures. Int. J. Pest Manag. 2017, 63, 364-370. [CrossRef]

75. Noor-ul-Ane, M.; Mirhosseini, M.A.; Crickmore, N.; Saeed, S.; Noor, I.; Zalucki, M.P. Temperature-dependent development of Helicoverpa armigera (Hübner) (Lepidoptera: Noctuidae) and its larval parasitoid, Habrobracon hebetor (Say) (Hymenoptera: Braconidae): Implications for species interactions. Bull. Entomol. Res. 2018, 108, 295-304. [CrossRef]

76. Oluwafemi, A.R.; Rao, Q.; Wang, X.Q.; Zhang, H.Y. Effect of Bacillus thuringiensis on Habrobracon hebetor during combined biological control of Plodia interpunctella. Insect Sci. 2009, 16, 409-416. [CrossRef]

77. Mathew Isaac, L.; Deepak, S.; Tripathi, C.P.M. Effect of Bacillus thuringiensis on oviposition and parasitization by parasitoid Habrobracon hebetor Say (Hymenoptera: Braconidae) on Corcyra cephalonica Stainton (Lepidoptera: Pyralidae). Int. J. Sci. Res. Rev. 2018, 7, 439-452.

78. Sneh, B.; Gross, S.; Gasith, A. Biological control of Spodoptera littoralis (Boisd.) (Lep., Noctuidae) by Bacillus thuringiensis subsp. entomocidus and Bracon hebetor Say (Hym., Braconidae). Zeitschrift für angewandte Entomologie 1983, 96, 408-412. [CrossRef]

79. Poinar, G.O., Jr. Taxonomy and biology of Steinernematidae and Heterorhabditidae. In Entomopathogenic Nematodes in Biological Control; Gaugler, R., Kaya, H.K., Eds.; CRC Press: Boca Raton, FL, USA, 1990; pp. 23-61.

80. Mbata, G.N.; Shapiro-Ilan, D.I. Compatibility of Heterorhabditis indica (Rhabditida: Heterorhabditidae) and Habrobracon hebetor (hymenoptera: Braconidae) for biological control of Plodia interpunctella (Lepidoptera: Pyralidae). Biol. Control 2010, 54, 75-82. [CrossRef]

81. Rumbos, C.I.; Athanassiou, C.G. The use of entomopathogenic nematodes in the control of stored-product insects. J. Pest Sci. 2017, 90, 39-49. [CrossRef]

82. Rossoni, C.; Kassab, S.O.; De Souza Loureiro, E.; Pereira, F.F.; Costa, D.P.; Barbosa, R.H.; Zanuncio, J.C. Metarhizium anisopliae and Beauveria bassiana (Hypocreales: Clavicipitaceae) are compatible with Cotesia flavipes (Hymenoptera: Braconidae). Fla. Entomol. 2014, 97, 1794-1804. [CrossRef] 
83. Rossoni, C.; Pereira, F.F.; Kassab, S.O.; Rodrigues, A.; Barbosa, R.H.; Zanuncio, J.C. Development of Eulophidae (Hymenoptera) parasitoids in Diatraea saccharalis (Lepidoptera: Crambidae) pupae exposed to entomopathogenic fungi. Can. Entomol. 2016, 148, 716-723. [CrossRef]

84. Mahdavi, V.; Saber, M.; Rafiee-Dastjerdi, H.; Mehrvar, A. Susceptibility of the Hymenopteran parasitoid, Habrobracon hebetor (Say) (Braconidae) to the entomopathogenic fungi Beauveria bassiana Vuillemin and Metarhizium anisopliae Sorokin. J. Biol. Sci. 2013, 6, 17-20. [CrossRef]

85. Kryukov, V.Y.; Kryukova, N.A.; Glupov, V.V. Susceptibility of Galleria mellonella larvae to anamorphic entomopathogenic ascomycetes under envenomation and parasitization by Habrobracon hebetor. Russ. J. Ecol. 2013, 44, 89-92. [CrossRef]

86. Kryukov, V.Y.; Kryukova, N.A.; Tyurin, M.V.; Yaroslavtseva, O.N.; Glupov, V.V. Passive vectoring of entomopathogenic fungus Beauveria bassiana among the wax moth Galleria mellonella larvae by the ectoparasitoid Habrobracon hebetor females. Insect Sci. 2018, 25, 643-654. [CrossRef] [PubMed]

87. Suma, P.; Amante, M.; Bella, S.; La Pergola, A.; Russo, A. Stored-product insect natural enemies in wheat industry in Sicily. IOBC-WPRS Bull. 2014, 98, 227-233.

88. Johnson, J.A.; Valero, K.A.; Hannel, M.M.; Gill, R.F. Seasonal occurrence of post-harvest dried fruit insects and their parasitoids in a culled fig warehouse. J. Econ. Entomol. 2000, 93, 1380-1390. [CrossRef]

89. Eliopoulos, P.A.; Athanassiou, C.G. Seasonal occurrence of dried fig pests and their parasitoids in a fig warehouse in Greece. IOBC-WPRS Bull. 2004, 27, 159-171.

90. Prozel, S.; Schöller, M. Five years of biological control of stored-product moths in Germany. In Advances in Stored Product Protection; Credland, P.F., Armitage, D.M., Bell, C.H., Cogan, P.M., Highley, E., Eds.; CABI Publishing: Wallingford, UK, 2003; pp. 322-324.

91. Trematerra, P.; Oliviero, A.; Savoldelli, S.; Schöller, M. Controlling infestation of a chocolate factory by Plodia interpunctella by combining mating disruption and the parasitoid Habrobracon hebetor. Insect Sci. 2017, 24, 503-510. [CrossRef]

92. Brower, J.H.; Press, J.W. Interaction of Bracon hebetor (Hymenoptera: Braconidae) and Trichogramma pretiosum (Hymenoptera: Trichogrammatidae) in Suppressing Stored-Product Moth Populations in Small Inshell-Peanut Storages. J. Econ. Entomol. 1990, 83, 1096-1101. [CrossRef]

(C) 2019 by the authors. Licensee MDPI, Basel, Switzerland. This article is an open access article distributed under the terms and conditions of the Creative Commons Attribution (CC BY) license (http:/ / creativecommons.org/licenses/by/4.0/). 